\title{
LECTURAS DE LA CRISIS EN CLAVE FEMINISTA: UNA COMPARACIÓN DE LA LITERATURA EN TORNO A LOS EFECTOS ESPECÍFICOS SOBRE LAS MUJERES
}

\section{READINGS FOR THE CRISIS IN FEMINIST KEY: A COMPARISON OF LITERATURE REGARDING THE SPECIFIC EFFECTS ON WOMEN}

\author{
ASTRID AGENJO CALDERÓN \\ Universidad Complutense de Madrid \\ astridagenjo@gmail.com \\ Fecha recepción: 27 de septiembre de 2011 \\ Fecha aceptación: 6 de diciembre de 2011
}

\begin{abstract}
RESUMEN
El presente texto trata de identificar cómo afecta la crisis de forma específica a las mujeres, entendiendo que ello depende de la diferente posición que éstas ocupan en el sistema socioeconómico respecto a los hombres, fruto del desigual poder del que disponen. Para ello, se recurre a una comparación de la literatura especializada en situar dichas relaciones de poder entre géneros como ámbito privilegiado de atención teórica; esto es, la literatura procedente de la Economía Feminista, diferenciando dos corrientes: la Economía Feminista de la Conciliación y la Economía Feminista de la Ruptura, tratando de identificar las principales similitudes y diferencias en el análisis que éstas realizan de la crisis y de sus efectos.
\end{abstract}

PALABRAS CLAVE: economía feminista, crisis económica, crisis de los cuidados

JEL: B54

\section{ABSTRACT}

This paper seeks to identify how the crisis affects specifically to women, understanding that this depends on the different position they take in the socioeconomic system with respect to men, as a result of unequal power they have. For this, we use a comparison of the literature specialized in placing those power relations between genders as priority area of theoretical attention: this is, literature from Feminist Economics, differentiating between two streams Feminist Economics of the Conciliation and Feminist Economics of the Ruptureand trying to identify the main similarities and differences in the analysis that they conducted in the crisis and its effects.

KEYWORDS: Feminist Economics, Economic Crisis, Care Crisis. 
Agenjo, Astrid. Lecturas de la crisis en clave feminista: una comparación de la literatura en torno a los efectos específicos sobre las mujeres.

"Simplemente haciendo encajar a las mujeres, sin cambiar las reglas del juego, significaría la mera deificación de las condiciones existentes de desigualdad social'

Braidtotti y Butler, 1997.

\section{INTRODUCCIÓN}

Actualmente nos encontramos inmersas en una situación de crisis profunda que amenaza el tejido social y el bienestar de las personas, poniendo en serio peligro las condiciones de vida de los sectores más vulnerables de la población ${ }^{1}$. No obstante, la gran mayoría de los análisis en torno a la crisis aquejan una ceguera respecto a los procesos de reproducción social, de forma que tales efectos pasan completamente desapercibidos.

Por este motivo, desde diferentes visiones críticas de la economía se están llevando a cabo análisis que tratan de denunciar ese sesgo de los discursos convencionales ${ }^{2}$, explorando cómo afecta la crisis a las condiciones de vida de sujetos diferencialmente posicionados en el sistema socioeconómico por razón de clase, raza o género entre otras. Esta última categoría es la que adquiere una relevancia fundamental en el presente estudio dado que el objetivo que se persigue es analizar cómo afecta la crisis de forma específica a las mujeres ${ }^{3}$.

Para ello se ha recurrido a una revisión de la literatura especializada, es decir, aquella que otorga a las relaciones de género una relevancia económica fundamental: la Economía Feminista (EF). Los análisis que se han seleccionado son aquellos que estudian el impacto de la crisis actual atendiendo tanto a la situación específica de las mujeres como a las diferencias entre mujeres y hombres, entendiendo que tales diferencias no son fruto de condiciones naturales o biológicas sino que son producto de una construcción cultural y de desigualdades de poder. Obviamente no se trata de ofrecer un discurso victimista sobre "cuánto peor están las mujeres" tras la crisis sino de comprender cómo ésta les afecta de forma diferenciada debido a la desigual posición que ocupan en el sistema socioeconómico.

La herramienta fundamental para reflexionar en torno a las ideas de cada uno de los análisis seleccionados nos la proporciona la clasificación realizada por Amaia Pérez Orozco en relación a las corrientes de pensamiento económico feminista (Pérez, 2006). La autora utiliza como criterio clasificador el grado de modificación de los paradigmas económicos androcéntricos, distinguiendo así entre la Economía Feminista de la Conciliación (EFC) y la Economía Feminista de la Ruptura (EFR).

\footnotetext{
${ }^{1}$ Extraído de las conclusiones del III Congreso de Economía Feminista celebrado en Baeza, abril de 2009. http://www.upo.es/congresos/economiafeminista

${ }^{2}$ Véase, por ejemplo, los semimonográficos y notas sobre la crisis en los números 7, 8, 9 y 10 de la Revista de Economía Crítica.

${ }^{3}$ El hecho de que sea el género el punto de vista elegido para mirar al mundo no implica que dejemos de insistir en la pertinencia de cruzarlo con el resto de categorías mencionadas a fin de reflejar las desigualdades sociales de la forma más fiel posible.
} 
Agenjo, Astrid. Lecturas de la crisis en clave feminista: una comparación de la literatura en torno a los efectos específicos sobre las mujeres.

La primera corriente (EFC) se presenta como una reformulación del discurso androcéntrico con nuevos conceptos y métodos provenientes del feminismo, mientras que la segunda (EFR) propone una ruptura central. No obstante, es necesario señalar que, tanto de forma general como respecto a los efectos de la crisis, estos análisis no son excluyentes; obviamente existen diferencias conceptuales y metodológicas fundamentales, pero lo cierto es que se aprecia una constante retroalimentación entre ambos. Así, el propósito de este trabajo será precisamente identificar la raíz de tales diferencias y tratar de explorar sus similitudes y posibles complementariedades.

La estructura del documento es la siguiente: tras este primer apartado introductorio se presentan brevemente algunas consideraciones clave relativas a la Economía Feminista, exponiendo la pertinencia de considerar un análisis no androcéntrico en torno a la crisis pero reconociendo la parcialidad del mismo. En el tercer y cuarto apartado se llevará a cabo el análisis de la EFC y la EFR respectivamente, atendiendo en primer lugar a una presentación de sus rasgos esenciales para, posteriormente, centrarnos en el análisis específico que realizan de la crisis. En el quinto apartado se tratarán de reflejar las principales similitudes y diferencias halladas entre las corrientes, mientras que el sexto y último punto estará dedicado a las reflexiones finales al respecto de los resultados obtenidos como consecuencia del análisis comparativo.

\section{LA ECONOMÍA FEMINISTA: ALGUNAS CONSIDERACIONES CLAVE}

Los estudios feministas en economía han supuesto, desde sus inicios, un cuestionamiento constante sobre cómo se ha construido la disciplina, permitiendo sacar a la luz la doble exclusión a la que han sido sometidas las mujeres en tanto que sujeto y objeto de estudio ${ }^{4}$. Esta ausencia de las mujeres en la economía ha estado socialmente condicionada por factores de género, lo cual ha tenido un gran impacto sobre los problemas tratados, los métodos planteados y los resultados obtenidos. De esta forma se ha impuesto una visión androcéntrica que no incluye "lo femenino" y que considera que los problemas que afectan a los hombres como grupo humano pueden extenderse al resto de la humanidad, sin dar cabida a lo problemático para las mujeres, sus actividades, sus usos del tiempo, sus modelos de percibir y sentir, y las lecturas que hacen de la realidad (Durán, 2000).

Entre tales enfoques androcéntricos se encuentran discursos de muy diversa índole -neoclásicos, institucionalistas, marxistas- y muy diferentes en cuanto a su epistemología, metodología y conclusiones políticas, pero todos coinciden en no otorgar significancia económica relevante a las relaciones de género y en

\footnotetext{
4 Los estudios feministas en economía se desarrollan casi en paralelo al conjunto del pensamiento económico. Si bien, es a partir de los años setenta cuando se inicia con fuerza una importante elaboración teórica y empírica. La creación de la Internacional Association For Feminist Economics (IAFFE) en 1992 y la publicación de su correspondiente revista "Feminist Economics" desde 1995 ha supuesto un empuje definitivo. Los desarrollos de la economía feminista de las últimas décadas se recopilan en obras como en Gardiner 1997; Carrasco 1999; Benería 2003 o Pérez 2006.
} 
Agenjo, Astrid. Lecturas de la crisis en clave feminista: una comparación de la literatura en torno a los efectos específicos sobre las mujeres.

la utilización de un discurso dicotómico basado en la invisibilización de las esferas económicas feminizadas, asociadas a lo no monetizado.

Dicha invisibilización responde al progresivo proceso de reducción del objeto de estudio de la economía al ámbito mercantil; un proceso que no ha sido neutral ante el género puesto que a medida que ciertas dimensiones se han constituido como económicas, a su vez también se han masculinizado. Por el contrario, las dimensiones calificadas como no económicas "se han identificado con los roles, espacios, intereses y características que históricamente se han asignado a las mujeres, en un proceso de dicotomización analítica, espacial y normativa, donde la creación de esferas separadas para hombres y mujeres en cada una de las dimensiones se ha retroalimentado" (Pérez, 2006:55). El concepto de trabajo ha sufrido este mismo estrechamiento progresivo, pasando a identificarse exclusivamente con el empleo remunerado que se realiza en el ámbito del mercado y quedando invisibilizadas el resto de actividades (Folbre y Hartmann, 1988, Carrasco et al., 2004 y 2009, Pérez, 2006).

Este acortamiento de los conceptos de economía y trabajo por parte del discurso convencional ha dado lugar a unos esquemas interpretativos que ofrecen una visión desfigurada de la realidad (Hartmann, 1981, Folbre y Hartmann, 1988, Pujol, 1992) porque, como señala Carrasco (2009:174): "esconden una parte importante de los procesos fundamentales para la reproducción social y humana -básicamente el trabajo que se realiza en los hogares - sin los cuales el mercado ni siquiera podría subsistir. Así, las teorías económicas, al excluir del cuadro analítico general el proceso de reproducción social de la población, consideran normalmente las condiciones de vida como un efecto final de la producción, adaptables a los procesos de acumulación".

No obstante, no todos los enfoques sensibles a las diferencias de género cuestionan tales sesgos androcéntricos: es decir, hay enfoques que tratan de insertar a las mujeres en el análisis pero sin cuestionar los marcos preexistentes, y hay enfoques cuyo punto de partida es precisamente la crítica y modificación de tales marcos. Esto supone una diferencia fundamental entre la Economía de Género y la Economía Feminista (Pérez, 2006).

\subsection{Economía de género}

Este enfoque utiliza como estrategia básica la calificada como "añada mujeres y revuelva"5. Se denomina economía de género porque "es la nomenclatura que mayoritariamente utilizan quienes pretenden seguir elaborando un discurso despolitizado y objetivista, frente a la adjetivación de feminista, que supone la asunción de un compromiso político explícito" (Ibíd.:11). Dentro de este enfoque se reconocen dos metodologías diferentes:

\footnotetext{
${ }^{5}$ Harding $(1986,1987)$ califica esta estrategia como "añada mujeres" y Hewitson (1999) la complementa como "añada mujeres y revuelva".
} 
Agenjo, Astrid. Lecturas de la crisis en clave feminista: una comparación de la literatura en torno a los efectos específicos sobre las mujeres.

- Análisis de "la mujer" como sujeto de estudio: atienden a la ausencia o subrepresentación de las mujeres como sujeto epistemológico en la economía. ${ }^{6}$ Harding (1986) los denomina como "Estudios de equidad".

- Análisis de "la mujer" como objeto de estudio o Empirismo feminista: se considera que la exclusión de las mujeres ha sido consecuencia de una mala aplicación del método científico; es decir, los enfoques androcéntricos han hecho mala ciencia porque no han atendido a la experiencia femenina. Por tanto, la solución que se plantea es insertar a las mujeres (como colectivo con una experiencia de opresión común) en los marcos teóricos preexistentes, aunque sin cuestionarlos. Su recuperación del género y las experiencias femeninas se da, por tanto, en los límites de lo que los enfoques androcéntricos consideran económico (el ámbito monetizado) y se centran fundamentalmente en el análisis de la participación femenina en el mercado laboral ${ }^{7}$.

\subsection{Economía Feminista}

La economía feminista considera que los enfoques androcéntricos lo son porque los propios criterios epistemológicos en los que se basan estaban sesgados, de ahí que se considere como tarea fundamental redefinir tanto los criterios de validación del conocimiento como el propio objeto de estudio de la economía (Ferber y Nelson, 1993 y 2003; Humphries, 1995; Kuiper y Sap, 1996; Hewitson, 1999). A partir de este cuestionamiento, se aspira a renovar la disciplina utilizando categorías analíticas y de interpretación que posibilitan una manera de interpretar la realidad económica en la que las mujeres no están ausentes.

Así, junto a esta crítica metodológica y epistemológica a los enfoques androcéntricos, desde la Economía Feminista se ha venido desarrollando una amplia temática en las últimas décadas. Tal y como se recoge en Carrasco (2009: 3), algunas de las cuestiones fundamentales que se han tratado son las siguientes: "la crítica al pensamiento económico clásico por no considerar en sus estudios el trabajo de las mujeres, la discusión sobre el concepto de trabajo, las características y funciones del trabajo doméstico, distintos aspectos de la participación y discriminación laboral de las mujeres, las políticas económicas y sus efectos diferenciados por sexo, los problemas de género y desarrollo y, más recientemente, los efectos de la liberalización comercial y los flujos monetarios sobre el trabajo y condiciones de vida de las mujeres, la invisibilidad de las mujeres en los modelos macroeconómicos, el sesgo masculino presente en los ingresos y gastos de los presupuestos públicos, la crítica y nuevas propuestas a las estadísticas y a la contabilidad nacional por no incluir los trabajos no remunerados, los estudios sobre usos del tiempo que permiten constatar las diferencias de dedicación a los distintos trabajos entre mujeres y hombres y el mayor tiempo total de trabajo realizado por las mujeres y el desarrollo de nuevos enfoques que permitan el análisis global de la

\footnotetext{
${ }^{6}$ El Comité sobre el Estatus de las Mujeres en la Profesión Económica realiza un informe anual sobre esta materia (Committee on the Status of Women in the Economics Profesión, www.cswep.org)

7 Ejemplos de esta perspectiva son: Jacobsen, 1994; Humphries (ed.), 1995; Dijkstra y Plantenga (eds.), 1997; Alles, 2000.
} 
Agenjo, Astrid. Lecturas de la crisis en clave feminista: una comparación de la literatura en torno a los efectos específicos sobre las mujeres.

sociedad manteniendo como objeto central la sostenibilidad de la vida humana, el bienestar y la calidad de vida de las personas".

Las reclamaciones que subyacen a estos análisis son, en primer lugar, que las mujeres también son capaces de crear conocimiento y elaborar discurso económico. $Y$, en segundo lugar, que son agentes sociales activas $y$, por tanto, es necesario que la economía estudie su presencia como tales. Pero es aquí donde surgen dos cuestiones fundamentales: ¿Quién es ese sujeto "la mujer" cuya experiencia económica (en cualquiera de las temáticas mencionadas) buscamos conocer? ¿Y cómo vamos a llevar a cabo el estudio de dicha experiencia?

Esas preguntas van a ser claves a la hora de comprender las diferencias entre corrientes económicas feministas. Siguiendo la terminología de Amaia Pérez Orozco (2006) distinguiremos entre la Economía Feminista de la Conciliación (EFC) y la Economía Feminista de la Ruptura $(E F R)^{8}$. Respecto a esta clasificación, es necesario señalar que, tal y como afirma Pérez (2006:7) "no se pretende encasillar a cada autora, sino proporcionar herramientas para debatir en torno a las ideas. En segundo lugar, no se pretende ni puede ofrecerse una visión de linealidad progresiva y/o homogénea del pensamiento económico feminista (...) Todo ello se relaciona con la tercera cuestión a resaltar, a saber: que la clasificación propuesta es una clasificación localizada en un tiempo y lugar concreto. Esto significa que es mutable y que no puede extrapolarse".

Esta diferenciación es la que servirá de base en este estudio sobre los efectos de la crisis, por lo que, una vez establecidas las cautelas pertinentes, trataremos de desentrañarla en los siguientes apartados.

\section{ECONOMÍA FEMINISTA DE LA CONCILIACIÓN}

Dentro del término general de economía feminista de la conciliación pueden diferenciarse distintos enfoques que, a pesar de sus diferencias -en gran medida ligadas a las distintas corrientes económicas androcéntricas de las que parten- dan como resultado un cuerpo teórico relativamente homogéneo ${ }^{9}$.

El rasgo común que se identifica en los enfoques de la EFC es que consideran que el análisis de la situación de las mujeres y de las relaciones de género no puede ser añadido sin cuestionar los marcos preexistentes. Para ello, y desde un punto de vista metodológico, deconstruyen los conceptos fundacionales de economía y trabajo utilizando dos estrategias que Pérez (2006) identifica como "conciliación" y "reversión de jerarquías" respectivamente. El primer paso común a ambas es la visibilización del "otro" oculto y ajeno a lo que

\footnotetext{
8 Otros textos que proporcionan este tipo de análisis comparativos son: Beasley, 1994, Humphries, 1995; Nelson, 1995; Carrasco, 1999 y 2005; Hewitson, 1999; Peterson y Lewis, 1999; Robeyns, 2000; Schneider y Shackelford, 2001; Agarwal, 2004; Benería, 2004; Picchio, 2005.

${ }_{9}$ Desde el feminismo marxista con el denominado debate sobre el trabajo doméstico (Benston, 1969; Morton, 1971) como desde el feminismo socialista anglosajón y las teorías de los sistemas duales (Young, 1980; Hartmann, 1979) o desde el enfoque producción-reproducción que parte de los enfoques radicales o reproductivos (Bryceson y Vuorela, 1984; Benería y Sen, 1987).
} 
Agenjo, Astrid. Lecturas de la crisis en clave feminista: una comparación de la literatura en torno a los efectos específicos sobre las mujeres.

habitualmente se considera el sistema económico, es decir, la esfera privadodoméstica (Véase, por ejemplo: Schneider y Shackelford, 2001).

Una vez visibilizada, se plantea la cuestión de cómo abordar el análisis económico del conjunto logrado esfera mercantil/esfera doméstica, es decir, qué importancia analítica se le debe conceder a cada una de ellas. En este punto las dos estrategias divergen: con la conciliación se optaría por otorgar el mismo valor analítico a los espacios escindidos, mientras que con la reversión de jerarquías se concedería mayor peso analítico al elemento que convencionalmente se había infravalorado: la esfera doméstica. Es necesario señalar que esta investigación se va a basar en aquellos análisis que utilizan la estrategia de la "conciliación", puesto que la "reversión de jerarquías" no ha sido una opción muy secundada por las economistas feministas y no existen análisis de la crisis lo suficientemente significativos ${ }^{10}$.

A nivel epistemológico, la propuesta que subyace es la del standpoint feminism (véase, entre otras: Harding, 1986/1996). Es decir, se plantea la necesidad de situar a "las mujeres" como sujeto homogéneo en la posición privilegiada del análisis, identificando nuevos problemas y atendiendo a los nuevos datos, relaciones y modelos que se generan.

El objetivo es, por tanto, revalorizar las actividades femeninas para lograr un reconocimiento público del significado de los trabajos de las mujeres para el sistema económico y de esta forma, uniéndolo a lo masculino ya valorado, obtener un conjunto más completo y humano (Pérez, 2006). Se trata, por tanto, de un análisis que denominamos como "integrador" y que trataremos de abordar con detalle en el presente apartado; primero de forma genérica y más tarde centrándonos en el caso concreto de la crisis y sus efectos

\subsection{Un análisis "integrador" de la economía}

El primer paso que se da en la EFC a la hora de analizar los procesos económicos, consiste en "descubrir" y visibilizar las tareas que han realizado las mujeres históricamente, para posteriormente poder analizarlas al mismo nivel que las actividades mercantiles y acabar así con su infravaloración.

Para ello, se propone el uso de una noción de trabajo más amplia basada en el denominado "principio del tercero"11, es decir, se considera que trabajo son aquellas actividades que podrían ser delegadas a una tercera persona. Este principio puede descomponerse en una serie de criterios definitorios que Himmelweit (1995: 4) resume en la siguiente cita: trabajo "es aquella actividad con un objetivo dado, que requiere un gasto de tiempo y energía, forma parte de una división social de las tareas y es separable de la persona que la realiza". Así, al tratarse de un criterio directamente derivado del mercado, el trabajo doméstico se valorará por su asimilación al trabajo mercantil

\footnotetext{
${ }^{10}$ La que se ha venido a denominar como "economía emocional" se distingue por aplicar esta segunda estrategia deconstructiva. Dos autoras fundamentales son: Delphy, 1970 y Beasley, 1994.

${ }^{11}$ Este criterio se expone por primera vez en Reid (1934), y es el mayoritariamente aceptado por la EFC.
} 
Agenjo, Astrid. Lecturas de la crisis en clave feminista: una comparación de la literatura en torno a los efectos específicos sobre las mujeres.

remunerado. El elemento que los distingue no va a ser el contenido o las tareas en sí mismas, sino las relaciones bajo las cuales se da la producción en uno y otro espacio $^{12}$.

Pero el interés para la EF radica en identificar el porqué de la invisibilización de este trabajo en el análisis económico convencional y las connotaciones de género implícitas; para ello en los análisis comienzan a usarse términos hasta entonces ajenos a la economía, y procedentes del feminismo tales como la División Sexual del Trabajo y/o la Familia Nuclear Tradicional.

- La división sexual del trabajo se trata de un concepto social, de origen marxista, que habla de estructuras colectivas e indica que el trabajo no se distribuye de modo neutral sino que mujeres y hombres tienen distintas responsabilidades sobre el trabajo doméstico y asalariado. Por tanto, su contenido transversal es la distribución estructural del trabajo en función del sexo y la adscripción de las mujeres a las tareas menos valoradas.

- El concepto de familia nuclear tradicional pretende describir la concreción micro de dicha estructura social en la familia como unidad básica de convivencia y decisión. Así, hace referencia a la división de roles hombre ganador de ingresos/mujer ama de casa como modo de organización social que ha ido de la mano de la desvalorización e invisibilización del trabajo doméstico y de una visión de la mujer como dependiente de los ingresos del hombre. Una estructura familiar que se desvela como elemento clave para que siga garantizándose la reproducción sin que los hombres vean alterada su disponibilidad para el mercado laboral.

A partir de la inclusión de estos conceptos en el análisis convencional, se logra desvelar que "esa caja negra de la categoría inactividad utilizada por las estadísticas de participación económica habituales escondía una ingente cantidad de tiempo de trabajo realizado por las mujeres. Es más, se descubre que las mujeres están doblemente presentes en la economía" (Pérez, 2006: 109).

Llegados a este punto, se plantea la cuestión de cómo abordar el análisis económico del conjunto logrado esfera mercantil/esfera doméstica, es decir, qué importancia analítica se le debe conceder a cada una de ellas. Como comentamos anteriormente, aquí las dos estrategias de deconstrucción divergen ("conciliación" y "reversión de jerarquías"); no obstante, como ya se ha dicho, en el presente trabajo nos vamos a ocupar exclusivamente de la estrategia de la "conciliación", a partir de la cual las esferas mercantil y doméstica se integran en un análisis global y completo, donde se les concede el mismo valor y estatus analítico. Pero para profundizar en la forma en que se lleva a cabo dicho análisis nos serviremos de un ejemplo concreto: la crisis y su impacto sobre las mujeres.

12 En el caso del ámbito privado-doméstico estaríamos hablando de imposibilidad de especialización y de incremento de la productividad, inexistencia de competencia, libertad de ritmos y horarios para realizar el proceso de trabajo, etc. (Pérez, 2006). 
Agenjo, Astrid. Lecturas de la crisis en clave feminista: una comparación de la literatura en torno a los efectos específicos sobre las mujeres.

\subsection{El análisis de la crisis desde la EFC}

Existe una amplia literatura sobre los efectos que las recesiones han tenido sobre las mujeres en términos de intensificación de su tiempo de trabajo no remunerado (i.e. Rubery, 1988; Tinker (ed.), 1990; Amott, 1993; Bakker (ed.), 1994; Villota (ed.), 1999).

En lo que respecta a la crisis iniciada en 2007, se entiende que ha sido una crisis financiera global cuya principal expresión ha sido la caída de la demanda global de la economía (Alberdi, 2009; Antonopoulos, 2009; CSW, 2009; Gálvez y Torres, 2009 y 2010; Larrañaga, 2009; OIT, 2009, 2011; Pazos, 2009; Seguino, 2009; Walby, 2009).

Este hecho ha propiciado el incremento del desempleo y el empeoramiento de las condiciones laborales a nivel global. Pero desde la EFC se entiende que el efecto de la crisis sobre el trabajo de las mujeres no se refleja únicamente en las tasas de actividad, ocupación y desempleo, sino que también se considera necesario tener en cuenta cómo afecta la crisis a los trabajos no remunerados que se realizan en el hogar.

Por otra parte, y debido a las consecuencias en el ámbito financiero, también se presta una especial atención al efecto de la crisis sobre la autonomía financiera y el acceso al crédito de las mujeres, así como al efecto sobre éstas de lo que, siguiendo a Gálvez y Torres (2010) denominaremos los "daños colaterales de la crisis" -en referencia al impacto sobre la migración y la pobreza, la alimentación, la educación, la salud, y los servicios sociales de bienestar-; aspectos todos que se superponen de múltiples maneras y crean una vertiginosa caída en la calidad de vida de las mujeres, en particular de las mujeres pobres, migrantes y pertenecientes a minorías (CSW, 2009).

\subsubsection{El trabajo total de las mujeres}

\section{Diferente situación de partida.}

Tradicionalmente el acceso al empleo formal, al salario y a los derechos sociales que ello conlleva han sido privilegio de los hombres; la EFC ha captado este hecho en la división sexual del trabajo por la que los hombres asumen el rol de sustentador familiar, quedando las mujeres adscritas a las tareas menos valoradas, esto es, el trabajo doméstico no remunerado. Las implicaciones de este desigual reparto por género son evidentes en el mercado de trabajo, manifestándose en una menor tasa de actividad femenina y, por tanto, "en una menor posibilidad de autonomía financiera para las mujeres, menor posibilidad de acceder al bienestar material y a la libertad de toma de decisiones asociadas a la tenencia de salario en una sociedad de mercado" (Gálvez y Torres, 2010: 95).

Esta norma social patriarcal coarta la libertad de las mujeres a la hora de acudir al mercado laboral, puesto que se ven obligadas a dedicar una parte importante de su tiempo y energía al hogar. Por esta razón, las mujeres "activas" presentan menores tasas de ocupación, mayores tasas de parcialidad y mayor 
Agenjo, Astrid. Lecturas de la crisis en clave feminista: una comparación de la literatura en torno a los efectos específicos sobre las mujeres.

temporalidad en sus contratos. Como señalan Gálvez y Torres (ibíd.), esta mayor precariedad de las mujeres se debe a una doble segregación laboral: de carácter ocupacional u horizontal (diferente concentración de mujeres y hombres en los sectores productivos) y de carácter vertical (son ellas las que ocupan los empleos menos relevantes, de menor calidad y sujetos al llamado "techo de cristal").

Pero se entiende que la intensidad y discriminación con que las mujeres asumen el trabajo doméstico no remunerado en mayor medida que los hombres no sólo depende del predominio de tales valores patriarcales sino que, de modo directo, también depende de la existencia de medios materiales que posibiliten un reparto igualitario de los usos del tiempo. De esta forma, el foco de atención teórica se traslada desde el trabajo total hacia el tiempo total de trabajo. Un cambio de mirada que permite reflejar la doble presencia de las mujeres en el mercado y en el hogar, haciendo hincapié no en la acumulación de las dos jornadas, sino en la obligación que tienen de asegurar su presencia en ambos espacios de forma simultánea (Balbo, 1978; Bianchi, 1978; Picchio, 1992; Borderías y Carrasco, 1994; Floro, 1999); una presencia que se ha visto intensificada tanto en tiempo como en trabajo tras la crisis, y que trataremos de explicar a continuación.

\section{Impacto de la crisis sobre el trabajo asalariado/trabajo doméstico}

Si bien podría pensarse que, debido a las características anteriormente descritas acerca del empleo de las mujeres ellas se verán más afectadas tras la crisis, lo cierto es que el efecto diferenciado sobre el trabajo de mujeres y hombres no es generalizable porque éste dependerá del espacio económico, de los sectores productivos y de la segregación laboral por género que se haya llevado a cabo (Gálvez y Torres, 2009 y 2010; Seguino, 2009).

En el caso de los países desarrollados, los sectores más perjudicados por las fuertes caídas de demanda tras la crisis han sido sectores tradicionalmente masculinizados (construcción, industria de bienes duraderos, etc.) por lo que han sido los hombres los más afectados por el desempleo; por ello, al menos en principio, se produjo una disminución de la brecha entre la tasa de paro masculina y femenina. No obstante, el informe de la OIT sobre el Empleo global de las mujeres de marzo de 2009 señala que la tasa de pérdida de empleo entre los varones se desaceleró, mientras que esa tasa entre las mujeres seguía subiendo ${ }^{13}$.

Una de las explicaciones que se ofrecen ante este fenómeno es que, aunque las mujeres sean muy eficientes a nivel individual, presentan un grave problema a la hora de acceder al mercado laboral debido a que llevan en la frente el cartel de menos disponible como consecuencia de las responsabilidades de cuidado que se les ha asignado históricamente (Pazos, 2009). Así, en una situación de crisis en la que, tanto la incertidumbre empresarial como el excedente de oferta de trabajo han ido en aumento, los

\footnotetext{
${ }^{13}$ La tasa de desempleo femenina ha alcanzando hasta un 7,4\% en 2009, en comparación con el $7 \%$ para los varones (OIT, 2009)
} 
Agenjo, Astrid. Lecturas de la crisis en clave feminista: una comparación de la literatura en torno a los efectos específicos sobre las mujeres.

incentivos a la contratación de la mano de obra masculina son mucho mayores, en detrimento de la femenina.

Otra explicación al incremento del desempleo femenino en los países ricos se achaca a los recortes presupuestarios del sector público, ya que "son las mujeres las que mayoritariamente están empleadas en educación, salud y servicios sociales" (Seguido, 2009: 3). Unos recortes que, a su vez, inciden negativamente sobre las responsabilidades hogareñas y asistenciales de las mujeres: la disminución en los gastos sociales y en la provisión de bienes públicos lleva consigo un incremento en el número de horas necesarias para hacer frente a las necesidades familiares y, a pesar de que en esta crisis haya más hombres que pierden su empleo, éstos continúan sin hacerse cargo de más horas de trabajo doméstico, recayendo prácticamente en su totalidad sobre las mujeres.

Por su parte, en los países en desarrollo son principalmente las mujeres las que están sufriendo los efectos del descenso de actividad económica. Tal y como señala Buvinic (2009), mientras que algunas mujeres en estos países pueden estar "protegidas" de los efectos a corto plazo de la crisis porque no tienen acceso a los mercados mundiales, la gran mayoría de ellas están empleadas en la industria de exportación (textiles, calzado, conservas o electrónica) y en la agricultura de alto valor, motivo por el cual son más propensas a sufrir la pérdida directa de empleo derivada de la contracción del comercio mundial. En cualquier caso, se señala que en estos países las mujeres también suelen ser despedidas primero dado que los hombres son considerados tradicionalmente los principales sostenes de la familia (Seguino, 2009; Walby, 2009).

A consecuencia de la reducción del empleo y los salarios, y dentro lo que Buvinic (2009) califica como "una segunda ronda de impactos de la crisis", las mujeres se ven obligadas a adoptar múltiples estrategias de supervivencia en el ámbito doméstico y de subsistencia (con el consecuente gasto de tiempo y energía) y a intensificar, a su vez, la búsqueda de ingresos en las actividades del sector informal ${ }^{14}$.

A partir de todas estas consideraciones, se entiende que el principal efecto de la crisis ha sido la intensificación de tiempos y trabajos no remunerados que principalmente asumen las mujeres en el seno de los hogares, es decir, una intensificación del esfuerzo, del sacrificio y de las cargas de todo tipo que pesan sobre ellas y que dan lugar a una situación de mayor vulnerabilidad (Antonopoulos, 2009; Buvinic, 2009; Galvez y Torres, 2009, 2010).

\subsubsection{La autonomía financiera de las mujeres}

Se considera que la crisis iniciada en el verano de 2007 es más que una simple crisis hipotecaria; es una crisis financiera global que ha atacado al sistema

\footnotetext{
${ }^{14}$ La definición del trabajo informal que realiza la OIT se centra en la naturaleza del empleo en términos de falta de protección y la falta de regulaciones, así como menores ingresos y peores condiciones de trabajo, situación en la que se calcula se encuentran al menos el $60 \%$ de las mujeres trabajadoras, aunque con importantes variaciones regionales (OIT, 2009).
} 
Agenjo, Astrid. Lecturas de la crisis en clave feminista: una comparación de la literatura en torno a los efectos específicos sobre las mujeres.

económico en su conjunto. En este sentido se presta especial atención a los efectos que ha producido la contracción del crédito formal, lo cual ha tenido un mayor impacto sobre los hombres en términos cuantitativos puesto que son los principales usuarios; sin embargo, en términos cualitativos son las mujeres las que han tenido mayores complicaciones para acceder al crédito tras la crisis porque tienen que enfrentarse a múltiples obstáculos relacionados con prejuicios de género y porque "tienden a carecer de las garantías necesarias debido a la desventaja social en la que se hallan, a los menores ingresos salariales y a sus limitaciones en la posesión de propiedades" (Gálvez y Torres, 2009: 23).

En el análisis que se realiza de los países en desarrollo, se considera que son las mujeres las que mayoritariamente se han visto afectadas por la práctica congelación del sector microfinanciero (según datos del Banco Mundial las mujeres conforman el $80 \%$ de la clientela de los programas de microcréditos en estos países). Aunque este no es el único factor que perjudicaría la obtención de ingresos y la autonomía financiera de las mujeres en estos países: un elemento adicional sumamente importante ha sido la disminución de recursos procedentes del exterior como consecuencia de la ralentización del flujo de remesas que la población inmigrante envía desde los países donde trabajan y de las que dependen una importante franja de la población en sus países de origen (Buvinic, 2009).

Por tanto, el análisis de las remesas cobra una importancia fundamental debido al proceso de "feminización"15 al que se han visto sometidas como consecuencia del aumento del volumen total y la proporción relativa de mujeres inmigrantes (la mayor parte empleadas en la economía asistencial y en los hogares de los países desarrollados) y, por tanto, de remisoras de ingresos a sus países de origen (OIT, 2009).

\subsubsection{Daños colaterales de la crisis}

Siguiendo esta terminología utilizada por Gálvez y Torres (2010) se incluyen en el análisis otros aspectos de la crisis económica que han redundados en serios retrocesos en la consecución de los Objetivos de Desarrollo del Milenio. se entiende que el impacto no sólo se ha dejado sentir en el empleo y en los ingresos familiares como se ha comentado anteriormente, sino también en la ingesta de alimentos (ODM 1), en la educación de las niñas (ODM 2 y 3), la igualdad de género (ODM 3), la salud infantil y materna y el VIH / SIDA (ODM 4-6), el acceso al agua y saneamiento (ODM 7), y la AOD, el comercio y la deuda (ODM 8) (PNUD y UNIFEM, 2009).

Es decir, se ha demostrado a partir de anteriores crisis de similares características que en los países en desarrollo "suelen ser las mujeres las que primero salen de la escuela, las que renuncian en mayor medida a los cuidados sanitarios y a los beneficios de bienestar que pudieran haber tenido a su disposición antes de la crisis" (Gálvez y Torres, 2010: 123). Asimismo se

\footnotetext{
${ }^{15}$ Las mujeres constituyen al menos el 50 por ciento de los flujos de trabajadores migrantes africanos y latinoamericanos y hasta el 75 ó el 80 por ciento de los que provienen del Asia Meridional y el Sudeste Asiático (OIT 2009).
} 
Agenjo, Astrid. Lecturas de la crisis en clave feminista: una comparación de la literatura en torno a los efectos específicos sobre las mujeres.

considera que los efectos negativos sobre el bienestar se han visto aun más agravados en la crisis actual como consecuencia de la gran subida de los precios alimentarios que tuvo lugar en 2008 y que ha afectado a la dieta básica de cientos millones de personas, aumentando dramáticamente la inseguridad alimentaria en las economías más atrasadas del planeta.

Asimismo, se entiende que en una situación de subida de precios como la que se ha producido, las mujeres agricultoras se ven obligadas a intensificar su trabajo y dedicar menos tiempo al auto-cuidado así como al cuidado de las niñas/os de quienes se hacen cargo. A este respecto, desde la Organización Mundial de la Salud se ha señalado ${ }^{16}$ que la crisis provocaría entre 200.000 y 400.000 muertes infantiles más por año entre 2009 y 2015 como resultado de complicaciones surgidas del embarazo y el parto, la falta de alimentos, la inmunización o como consecuencia de una mala calidad del agua y la higiene.

La OMS también afirma que en etapas de crisis aumenta el estrés de las mujeres, sus enfermedades crónicas, la tendencia a la depresión o al alcoholismo como consecuencia de la pérdida de ingresos y, en general, por el empeoramiento de las condiciones de vida. Aunque, como señalan Gálvez y Torres (2010), hay que tener en cuenta que los hombres también se enfrentan a problemas específicos derivados de su incapacidad de seguir teniendo el rol de sustentador familiar, derivando en problemas de alcoholismo o suicidios 0 incluso en un aumento de la violencia que se ejerce contra las mujeres, tal y como ocurrió en crisis anteriores.

\section{ECONOMÍA FEMINISTA DE LA RUPTURA}

La EFR se caracteriza por su crítica a las corrientes de pensamiento económico androcéntrico y por el intento de trascender sus dicotomías fundacionales. En este sentido, se considera que la invisibilidad de los "otros" femeninos en el discurso convencional (como el trabajo doméstico, el hogar) no es un mero sesgo u olvido sino que se trata de un factor intrínseco para que exista "lo mismo", para que sea posible una estructura mercantil masculinizada. Por esta razón, desde un punto de vista metodológico, se apuesta por no conceder el mismo valor analítico a esferas económicas escindidas (mercantil/ doméstica), sino por centrarse en el elemento que las trasciende y las contiene: la sostenibilidad de la vida (Albelda, 1997; Benería, 1995; Kuiper y Sap, 1996; Nelson, 1996; Carrasco, 2001, 2009 y 2011; Picchio, 1999; Pérez, 2006; León, 2009).

La propuesta epistemológica que subyace no consiste en realizar un análisis abstracto de los procesos económicos cuyos resultados sean universalizables para todas "las mujeres" 17 , sino que lo que se propone es la construcción de conocimientos situados, "Situated Knowledges" (Haraway, 1991), es decir, afirmaciones históricas y culturalmente localizadas que busquen la

\footnotetext{
16 "Downturn could kill 400,000 children, warns Margaret Chan," New York Times, 14 de marzo de 2009.

17 Se considera que la noción del sujeto fuerte "las mujeres" es limitada en el sentido de que esa pretensión de sororidad universal (por compartir una condición común de opresión) es falsa, ya que existen múltiples diferencias entre ellas.
} 
Agenjo, Astrid. Lecturas de la crisis en clave feminista: una comparación de la literatura en torno a los efectos específicos sobre las mujeres.

visualización parcial de procesos concretos. En este sentido, para la EFR resulta difícil establecer el límite entre lo que es y no es economía, y lo que es y no es trabajo, puesto que se entiende que dicha frontera sólo tiene sentido de forma situada. Por esta razón, se apuesta por la interdisciplinariedad, haciendo una elección contextualizada y estratégica de los métodos, y sin establecer fronteras previas al análisis (Pérez, 2006).

Resulta evidente, por tanto, que la EFR es una corriente en pleno proceso de construcción y de exploración de nuevos campos y horizontes feministas. Nuestro objetivo en el presente apartado será exponer sus ideas clave detallando, en primer lugar, la transformación que llevan a cabo del discurso convencional, algo que resultará fundamental para, posteriormente, poder comprender el análisis que se hace de la crisis y sus efectos específicos sobre las mujeres.

\subsection{Un análisis "transformador" de la economía}

A partir del término sostenibilidad de la vida (SV) no se pretende dar una nueva definición cerrada de la economía, sino que se intenta ofrecer una solución abierta que trascienda la dicotomía de lo económico/no económico y cuyo objetivo central sea el cuidado de la vida. Como señala Carrasco (2009:183), se trata de:

\footnotetext{
"un concepto que representa un proceso histórico de reproducción social, un proceso complejo, dinámico y multidimensional de satisfacción de necesidades en continua adaptación de las identidades individuales y las relaciones sociales, un proceso que debe ser continuamente reconstruido, que requiere de recursos materiales pero también de contextos y relaciones de cuidado y afecto, proporcionados éstos en gran medida por el trabajo no remunerado realizado en los hogares [...]. Un concepto que permite dar cuenta de la profunda relación entre lo económico y lo social, que sitúa a la economía desde una perspectiva diferente, que considera la estrecha interrelación entre las diversas dimensiones de la dependencia y, en definitiva, que plantea como prioridad las condiciones de vida de las personas, mujeres y hombres".
}

Dado que la economía se entiende como el proceso de generación de recursos para satisfacer necesidades y la creación de condiciones para una vida digna de ser vivida, los mercados dejan de tener un lugar privilegiado en el análisis y pasan a integrarse a él de forma derivada, por el papel que juegan en el proceso de sostenibilidad de la vida. Es decir, en dicho proceso los mercados intervienen sólo parcialmente ya que la mayor parte se desarrolla en lo que algunas autoras definen como el "espacio de desarrollo humano" (Picchio, 1999, 2001 y 2005; Bosch, et al. 2005; Amoroso, et al. 2003; Carrasco, 2009; o Pérez, 2010). Este proceso queda ilustrado en la Figura 1.

En ella se plasma el hecho de que "las condiciones de vida de la población no dependen sólo del salario; éste representa una parte significativa en las sociedades que vivimos, ya que tener ingresos monetarios es absolutamente imprescindible; pero las posibilidades de vivir una vida en condiciones adecuadas dependen también de las posibles aportaciones del sector público y del trabajo no asalariado realizado desde los hogares. Las condiciones en que se realiza la actividad laboral en el mercado son importantes para la calidad de vida de los y las trabajadores/as, pero también son importantes las condiciones 
Agenjo, Astrid. Lecturas de la crisis en clave feminista: una comparación de la literatura en torno a los efectos específicos sobre las mujeres.

en que se desarrolla la vida cotidiana más allá del empleo, lo que incluye la organización de los tiempos, horarios, espacios y la carga total de trabajo doméstico y de cuidados para la o las personas que lo realizan" (Ibíd:212).

Figura 1: El circuito del trabajo.

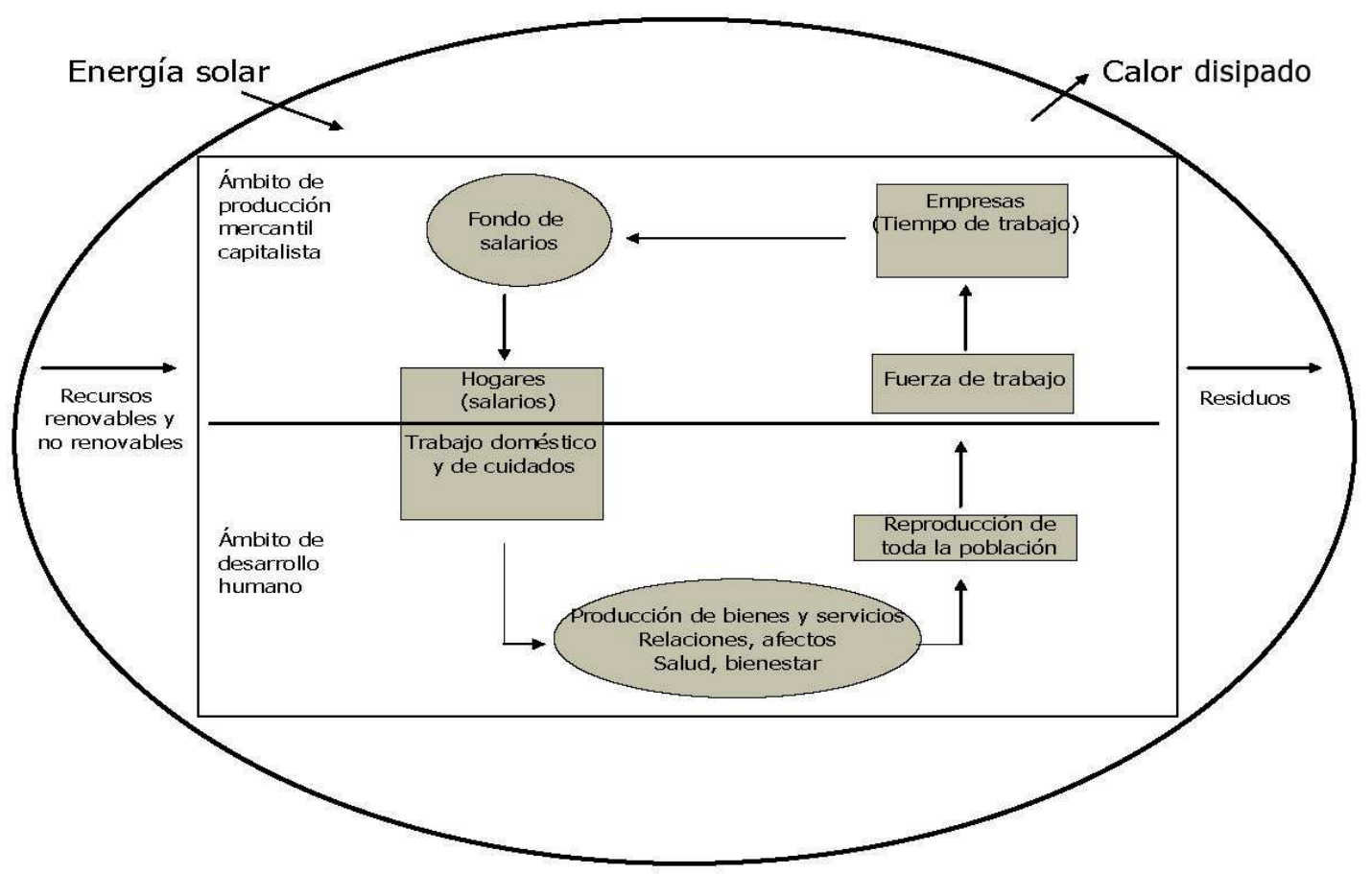

Fuente: Carrasco, C. (2011: 209).

Esta nueva visión de la economía se centra explícitamente en las personas, pero no en términos de agregación de individuos aislados sino en términos de aprovisionamiento social, evitando así caer en un estudio de las acciones y elecciones individuales. Como señala León (2009:1) "no se trata sólo de desplazar el control o las decisiones de un grupo hegemónico a otro, ni siquiera sólo de 'desprivatizar' esas decisiones, sino de afrontar transformaciones de fondo que lleven de una economía centrada en la acumulación y la tiranía del mercado, hacia una orientada a la sostenibilidad de la vida, la justicia y la democracia. Esto supone cambios en la matriz productiva, en las visiones y políticas acerca de quiénes y cómo hacen economía, de qué y cómo producir, qué y cómo consumir, de cómo, en última instancia, reproducir la vida".

Es decir, se considera que el resultado óptimo de los procesos económicos es la satisfacción social de necesidades y no tanto la felicidad individual, pero esta cuestión nos remite irremediablemente al debate sobre cuáles son esas necesidades que hay que satisfacer para "vivir una vida que merezca la pena". A pesar de la dificultad de establecer un consenso sobre ello ${ }^{18}$, sí se ha logrado cierto acuerdo en torno a la concepción multidimensional de las mismas y a su carácter endógeno respecto al sistema económico. En primer lugar, se considera que las necesidades incluyen dos dimensiones indisolublemente

\footnotetext{
${ }^{18}$ Este es un debate que tiene varios puntos de encuentro con el enfoque de las "capacidades". El no 9 (2/3) de Feminist Economics está dedicado a ello.
} 
Agenjo, Astrid. Lecturas de la crisis en clave feminista: una comparación de la literatura en torno a los efectos específicos sobre las mujeres.

ligadas: una "material" (aspectos como la alimentación, la protección del frio, etc.) y otra "inmaterial" (referida a las necesidades de afecto, cuidado, establecimiento de vínculos sociales, etc. en las que los aspectos personales y subjetivos tales como los cuerpos, el sexo o las emociones adquieren un peso analítico clave). En segundo lugar, las necesidades no se entienden como un elemento biológicamente determinado o exógeno al sistema económico sino que se considera que éstas se construyen y expresan socialmente y, por tanto, su definición se ve directamente afectada por las relaciones de poder.

La importancia que se otorga a las necesidades y a su carácter multidimensional permite un posible acercamiento tanto al ecologismo social o a enfoques como el buen vivir/vivir bien. El nexo con estas ramas de pensamiento se sitúa en la utilización de la SV como objetivo central; una sostenibilidad que "no sólo hace referencia a la posibilidad real de que la vida continúe -en términos humanos, sociales y ecológicos-, sino a que dicho proceso signifique desarrollar condiciones de vida, estándares de vida o calidad de vida aceptables para toda la población. Sostenibilidad que supone pues una relación armónica entre humanidad y naturaleza, y entre humanas y humanos" (Bosch et al., 2005: 322).

Por otra parte, se entiende que toda actividad que forme parte de este proceso de SV debería ser considerada al analizar el trabajo, el cual vendría definido como "la práctica de creación y recreación de la vida y de las relaciones humanas" (Ibíd.: 331). Esta definición recupera los elementos personales hasta entonces periféricos al análisis como la construcción de afectos y relaciones; asimismo se otorga una relevancia fundamental al hecho de que son cuerpos sexuados quienes lo llevan a cabo.

Es evidente que los límites de esta forma de entender el trabajo son vagos, pero no obstante se trata de una opción estratégicamente escogida puesto que posibilita el descentramiento de lo mercantil masculinizado. $Y$ si bien existe un importante debate sobre la conveniencia de concretar el término trabajo, la prioridad para las autoras es "buscar conceptos situados para describir fenómenos específicos" (Pérez, 2006: 175). Una opción que exige ampliar las metodologías de análisis -con el consecuente cruce de fronteras disciplinaresy elegir las herramientas en función de su utilidad en la provisión de ideas. Es decir, se elige "la metodología más apropiada en función del contexto y de las preguntas que han de contestarse", teniendo como resultado una economía "orientada a los problemas" y no "orientada al método" (Ibíd.: 177).

En relación al trabajo, desde la EFR se considera que ciertamente existe una tensión estructural entre éste y el capital, pero no obstante se sostiene que ese conflicto es más profundo puesto que se da "entre el capital y todos los trabajos... entre el capital y la vida" (Pérez, 2010: 135). Es decir, se entiende que existe un conflicto social entre la lógica del beneficio que subyace a la operación de los mercados y la lógica de la sostenibilidad de la vida. De ahí que algunas autoras hablen de una economía de iceberg" ${ }^{19}$ "porque hay dos

\footnotetext{
${ }^{19}$ Esta metáfora la utilizan autoras como Mies (1996) y Durán, quien afirma que "puede decirse que la economía española es como un iceberg, porque flota gracias a los dos tercios del esfuerzo colectivo que permanece invisible" (1999: 27).
} 
Agenjo, Astrid. Lecturas de la crisis en clave feminista: una comparación de la literatura en torno a los efectos específicos sobre las mujeres.

partes claramente diferenciadas (por la lógica que las mueve) y porque una ha de permanecer necesariamente oculta para sostener el sistema" (Ibíd.:136). Será sobre esta representación del iceberg sobre la que la EFR base su análisis del sistema socioeconómico y sobre la que estudie el impacto de los procesos económicos. Pero para profundizar en la forma en que se lleva a cabo dicho análisis nos serviremos de un ejemplo concreto: la crisis y su impacto sobre las mujeres.

\subsection{El análisis de la crisis desde la EFR}

Desde la EFR se caracteriza la crisis actual como una crisis "acumulada y multidimensional [...] que atraviesa todas las esferas no sólo del sistema, sino de la vida: una crisis civilizatoria" (Ibídem.). Por ello, la caída de la demanda global no se considera como la única ni la principal manifestación de la crisis ya que también se toman en cuenta otros desajustes que estaban poniendo en jaque al sistema incluso antes del estallido del colapso financiero: la crisis ecológica o la crisis de reproducción social entre otras (Carrasco, 2009 y 2011; Herrero, 2010; León, 2009; Pérez, 2010).

Para comprender el alcance y gravedad de esta situación en lo referente a las mujeres, se propone situar los cuidados como punto de vista estratégico desde el que analizar el sistema socioeconómico y los conflictos y desigualdades sociales de los que se sirve para persistir. Una nueva perspectiva que les permite profundizar en una dimensión de la crisis que consideran olvidada por el discurso convencional pero que afecta de forma específica a las mujeres: la crisis de los cuidados.

\subsubsection{La crisis de los cuidados}

\section{Los cuidados como punto de vista estratégico}

En la EFR la obtención de beneficios y el crecimiento económico pierden el privilegio de ser los elementos que organizan los tiempos, los espacios y la actividad humana; por el contrario, los ejes sobre los que se articula la sociedad son la reproducción social, la satisfacción de las necesidades y el bienestar humano (Herrero, 2010). Y será en torno a estas ideas desde donde se cuestionen los distintos trabajos, su relevancia y contenido. De esta forma, el tipo de trabajo que adquiere el protagonismo central en los análisis es aquel que permite a las personas crecer, desarrollarse y mantenerse como tales; un trabajo en el que "las mujeres no son personas secundarias y dependientes sino personas activas, actoras de su propia historia, creadoras de culturas y valores distintos a los del modelo capitalista y patriarcal" (Ibíd.: 9). Esto es, el trabajo de cuidados.

La EFR utiliza esta noción de trabajo de cuidados como ejemplo localizado y estratégico para pensar el sistema socioeconómico desde una perspectiva alternativa que descentre a los mercados capitalistas. Este concepto surge de las experiencias femeninas en el contexto occidental y trata de conectar las hasta ahora escindidas esferas del trabajo remunerado y no remunerado, haciendo referencia tanto a las tareas acometidas como a la lógica subyacente 
Agenjo, Astrid. Lecturas de la crisis en clave feminista: una comparación de la literatura en torno a los efectos específicos sobre las mujeres.

a la actividad. Obviamente, como señala Pérez (2010: 137), los cuidados no son "el todo" del sistema económico, pero sí representan una parte importante de las condiciones de vida al ocuparse de "la gestión y mantenimiento cotidiano de la vida y la salud, (al) hacerse cargo de los cuerpos sexuados atravesados por (des)afectos". Se les considera estratégicos "porque todas las personas los necesitamos en todos los momentos de nuestra vida, están absolutamente atravesados por la desigualdad de género, en gran medida se garantizan fuera de las esferas monetizadas de la economía y las interacciones entre las esferas no monetizadas y el mercado en este ámbito son complejas y cambiantes" (Ibíd.).

La desigualdad de género que atraviesa a los cuidados se pone de manifiesto al observar cómo éstos se han resuelto históricamente. Desde la EFR se sostiene que el modelo tradicional de reparto de los cuidados se ha basado, a nivel macro-social, en la división sexual del trabajo que asigna a las mujeres las tareas de cuidados y a los hombres el trabajo asalariado; y a nivel micro-social, en la imposición de la familia nuclear "hombre ganador del pan / mujer ama de casa" como norma. Estos dos ejes han atravesado no sólo la estructura del mercado laboral, sino también el estado del bienestar e incluso la propia construcción de las identidades. Esta desigual responsabilidad sobre los cuidados se ha forjado como una de las claves de la desigualdad entre mujeres y hombres y una de las principales fuentes de precariedad laboral de y/o mayor pobreza relativa de ellas (los cuidados implican una gran cantidad de tiempo y energía en el hogar de forma gratuita que se tienen que restar del tiempo disponible para otra actividad remunerada); por otra parte, genera enormes tensiones en las mujeres al intentar realizar los distintos trabajos simultáneamente (la doble presencia).

Este modelo de reparto de los cuidados configura la base oculta del iceberg económico sobre la que se sostienen las estructuras económicas y el sostenimiento de la vida humana. De forma que, a pesar de las gravosas consecuencias para las mujeres, garantiza "una aparente paz social" (Ibídem.) al negar legitimidad al conflicto básico capital-vida y hacerlo desaparecer en términos sociales y políticos. Pero dicho modelo ha entrado en quiebra en las últimas décadas en el contexto occidental y han salido a la luz las tensiones estructurales del sistema, dando lugar a lo que se ha denominado como la crisis de los cuidados.

\section{La crisis de los cuidados}

Los factores que, de forma general, han propiciado la ruptura del modelo tradicional de cuidados son, en primer lugar, el acceso generalizado de las mujeres al empleo que les ha proporcionado independencia económica y las ha convertido en sujetos políticos de pleno derecho. Así, el trabajo doméstico y de cuidados ha pasado a verse como "una atadura de la que hay que huir" (Herrero, 2010). Paralelamente se han establecido algunas transformaciones sociales que complican aun más la gestión de los cuidados. Nos referimos al envejecimiento de la población; al crecimiento urbano desbocado y la destrucción de espacios públicos; a la precarización de la vida que obliga a plegarse a los ritmos y horarios que impone la empresa; a la pérdida de redes 
Agenjo, Astrid. Lecturas de la crisis en clave feminista: una comparación de la literatura en torno a los efectos específicos sobre las mujeres.

sociales de apoyo (intergeneracionales, comunitarias...) que fuerza a resolver los asuntos cotidianos de una forma mucho más individualizada; y al progresivo desmantelamiento del Estado del bienestar y la privatización de ciertos servicios sociales que trataban de paliar algunos de estos problemas (Ibíd.).

Esta conjunción de factores hace más visible que nunca la necesidad de una redistribución social de las responsabilidades de cuidados entre las distintas instituciones de la economía, no sólo con la participación de los hogares sino también del Estado y de las empresas. Sin embargo, ni uno ni otras se están haciendo cargo del problema.

Las empresas han disminuido su responsabilidad social como consecuencia de la reducción de sus cotizaciones a la seguridad social y de la posibilidad de flexibilizar tiempos y espacios de trabajo (aunque paradójicamente han encontrado en la esfera de los cuidados una nueva oportunidad de negocio a través de la mercantilización y privatización de este tipo de servicios). Al privatizarse los cuidados, no sólo se han convertido en un "mecanismo multiplicador de desigualdades sociales" (Pérez, 2010: 138) sino que además las empresas se han apropiado de una parte importante de las obligaciones del Estado, quedando éste únicamente al cargo de determinadas ayudas y servicios sociales para casos concretos ${ }^{20}$. Si bien es cierto que se ha observado una tendencia al incremento de este tipo de prestaciones, desde la EFR se sostiene que son "estrategia(s) de parcheo que no implica(n) soluciones de fondo" (Ibíd.). De esta forma la resolución de la crisis ha vuelto a los hogares, y en ellos nuevamente a manos de las mujeres; son ellas quienes tienen que reorganizarse y hacer malabarismos con los recursos que tienen a su alcance: bien familiares (transferencia generacional del trabajo de cuidados de madres a abuelas) o bien monetarios (en caso de mujeres que por su condición de clase puedan comprar en el mercado parte de los trabajos de cuidados que demanda su familia), y viviendo su falta de atención con un fuerte sentimiento de culpa.

Las mujeres migrantes juegan un papel especialmente relevante en esta crisis de los cuidados que, como señala Carrasco (2009: 186), ya ha adquirido dimensiones globales "involucrando largas cadenas de mujeres organizadas sobre la base de ejes de poder, donde mujeres de mejor situación económica transfieren parte de sus cuidados a mujeres de posiciones más bajas". Es decir, las mujeres migrantes asumen en muchos casos trabajos de cuidados mal pagados dejando las responsabilidades de cuidados en sus países de origen en manos de otras mujeres (hermanas, abuelas, hijas mayores...). Estas cadenas globales de cuidados son el exponente visible de la exportación del problema, aunque, naturalmente, sin llegar a resolverlo (Ibíd.).

Así, se entiende que la crisis de los cuidados no se ha resuelto puesto que sigue viviéndose de manera individualizada y no politizada: "en lugar de buscarse soluciones sistémicas y colectivas, lo que encontramos es un doble proceso de redimensionamiento de la división sexual del trabajo a nivel global y

${ }^{20}$ Como señala Herrero (2010:6), son ayudas y servicios sociales que "sirven para gestionar una excepción: la de que no haya mujeres del entorno familiar que se puedan ocupar de cuidar". 
Agenjo, Astrid. Lecturas de la crisis en clave feminista: una comparación de la literatura en torno a los efectos específicos sobre las mujeres.

de reprivatización de la reproducción social (doble privatización porque sigue siendo responsabilidad del ámbito privado-doméstico, y porque cada vez se echa más mano de la compra de cuidados en el mercado) [...] (permaneciendo) inamovibles las coordenadas previas que daban cuerpo a un sistema insostenible e injusto" (Pérez, 2010: 139).

\subsubsection{La dimensión económica de la crisis}

Dado que desde la EFR la crisis actual se considera como multidimensional, también se considera necesario señalar los efectos que la recesión económica ha tenido sobre las mujeres de forma específica, los cuales van a venir derivados de su participación activa en los cuidados (Carrasco, 2010).

En primer lugar se señalan los efectos derivados de la disminución de ingresos en el hogar como consecuencia del desempleo y el empeoramiento de las condiciones laborales. En este sentido se destaca la intensificación del esfuerzo y el trabajo de las mujeres en el hogar -fundamentalmente en las mujeres de rentas más bajas- para afrontar esta disminución de ingresos y así evitar que el sostenimiento de la familia se vea perjudicado. Asimismo se considera que esta situación en el hogar puede impactar gravemente sobre las condiciones de vida de mujeres y niñas debido al incremento de la violencia contra ellas, al aumento de miedos, ansiedades e inseguridades, a la reducción de su tiempo libre, etc.

Si bien no se presta especial atención a los ingresos procedentes del sector financiero o del sector exterior, sí se hace mayor hincapié en las rentas procedentes del Estado. En este sentido se señalan los efectos derivados de la reducción del gasto social que incrementa el trabajo de las mujeres en distintas áreas, como el cuidado en la salud, en la educación, etc.; y se señala con especial preocupación las repercusiones en los sistemas de pensiones de las mujeres.

Por último se identifican otros "daños colaterales" como el aumento en las migraciones femeninas (que da lugar a las cadenas globales de cuidados); el incremento de los niveles de pobreza y la disminución de la calidad de vida, "particularmente, de mujeres que encabezan una tipología de hogares que ha estado en continuo aumento, los hogares unipersonales o monoparentales femeninos" (Ibíd.: 16). A partir de tales consideraciones, se advierte del riesgo que supone la crisis económica en el contexto actual de crisis de los cuidados, ya que es un momento en el que es fácil replegarse a posiciones defensivas del empleo y de lo productivo, ensalzando la economía "real" frente a la financiera, perdiendo de vista que la primera es tan propia del sistema patriarcal como la segunda (Pérez, 2010).

\section{EL ANÁLISIS COMPARATIVO ENTRE LA EFC Y EFR RESPECTO A LA CRISIS}

El objetivo de la presente investigación ha sido analizar cómo afecta la crisis de forma específica a las mujeres. Para responder a esta cuestión se ha recurrido a una comparación de la literatura especializada en situar las relaciones de 
Agenjo, Astrid. Lecturas de la crisis en clave feminista: una comparación de la literatura en torno a los efectos específicos sobre las mujeres.

poder entre géneros como ámbito privilegiado de atención teórica; esto es, la literatura procedente de la Economía Feminista, diferenciando para ello dos corrientes: la EFC y la EFR. A continuación presentamos las principales similitudes y diferencias que se han hallado tras el análisis comparativo.

Una de las primeras reflexiones surgidas tras este estudio es que ciertamente podemos considerar la EF como una rama de pensamiento económico propia. No se trata simplemente de la adición de la variable "género" al discurso convencional, sino que conlleva una manera distinta de analizar los procesos económicos, utilizando conceptos y herramientas particulares que tratan de socavar las relaciones de desigualdad por género; una desigualdad que tanto la EFC como la EFR entienden no procede de diferencias biológicas entre mujeres y hombres, sino que es consecuencia de la diferente capacidad de actuación y poder del que disponen. El consenso en torno a esta cuestión es la primera similitud que encontramos entre las dos corrientes analizadas. Aunque no es la única.

Tanto la EFC como la EFR tratan de recuperar los elementos femeninos invisibilizados, revalorizando las actividades que históricamente han realizado las mujeres y difuminando las imágenes androcéntricas que se dan de ellas. De ahí que ambas corrientes utilicen conceptos básicos dentro de la EF como la división sexual del trabajo y la familia nuclear tradicional.

Por último, las dos corrientes pretenden transformar una realidad que se considera injusta y tratar así de mejorar las condiciones de vida de las mujeres: ¿por qué iban a tratar de visibilizar las desiguales condiciones en que afecta la crisis a las mujeres si no es para hacer algo al respecto? No obstante, cada una de ellas lleva a cabo esta apuesta teórica y política de un modo diferente, lo cual se ve reflejado en el análisis que cada corriente hace de la crisis y de sus efectos específicos sobre las mujeres. Unas diferencias que trataremos de abordar a continuación y que podemos resumir en la siguiente tabla:

Tabla 1: Diferencias entre la EFC y la EFR

\begin{tabular}{|l|l|l|}
\hline Diferencias halladas: & EFC & EFR \\
\hline \begin{tabular}{l|l} 
Visión de los procesos \\
económicos
\end{tabular} & $\begin{array}{l}\text { Análisis integrador de la } \\
\text { economía mediante la } \\
\text { conciliación de los términos } \\
\text { dicotómicos: mercado/hogares } \\
\text { Se centran en los impactos en el } \\
\text { ámbito mercantil y el ámbito } \\
\text { doméstico al mismo nivel }\end{array}$ & $\begin{array}{l}\text { Análisis transformador de la } \\
\text { economía mediante la } \\
\text { trascendencia de las } \\
\text { dicotomías. Se analiza la } \\
\text { crisis por su impacto en los } \\
\text { procesos de sostenibilidad } \\
\text { de la vida }\end{array}$ \\
\hline Metodología de análisis & $\begin{array}{l}\text { Se compaginan métodos } \\
\text { convencionales con otros } \\
\text { procedentes del análisis } \\
\text { feminista }\end{array}$ & $\begin{array}{l}\text { Se utilizan conceptos } \\
\text { transversales a la } \\
\text { "economía" y el "trabajo" }\end{array}$ \\
\hline
\end{tabular}

Fuente: Elaboración propia 
Agenjo, Astrid. Lecturas de la crisis en clave feminista: una comparación de la literatura en torno a los efectos específicos sobre las mujeres.

\section{Visión de los procesos económicos}

En la EFC se analiza la situación de crisis actual otorgando el mismo valor a la esfera doméstica y a la esfera mercantil. Esta visión "integradora" de los procesos económicos se debe a la utilización de una estrategia concreta basada en la conciliación de los términos dicotómicos preexistentes. Unos términos que pueden integrarse porque previamente se ha modificado su carga valorativa: ahora los elementos feminizados del ámbito doméstico, antes ocultos, tienen el mismo valor analítico que los mercantiles masculinizados.

Por su parte, la EFR no pretende analizar la crisis atendiendo a los efectos sobre la esfera doméstica y mercantil al mismo nivel, sino que se centra en el impacto de la crisis sobre el elemento que las trasciende y las contiene, esto es, la sostenibilidad de la vida. Este término bisagra hace referencia a los procesos de satisfacción de las necesidades en el que interviene tanto la producción mercantil como la doméstica, de forma que para analizar el efecto de la crisis se propone establecer un punto de vista que incorpore ambos aspectos: el cuidado de la vida.

La siguiente figura trata de exponer las diferencias comentadas:

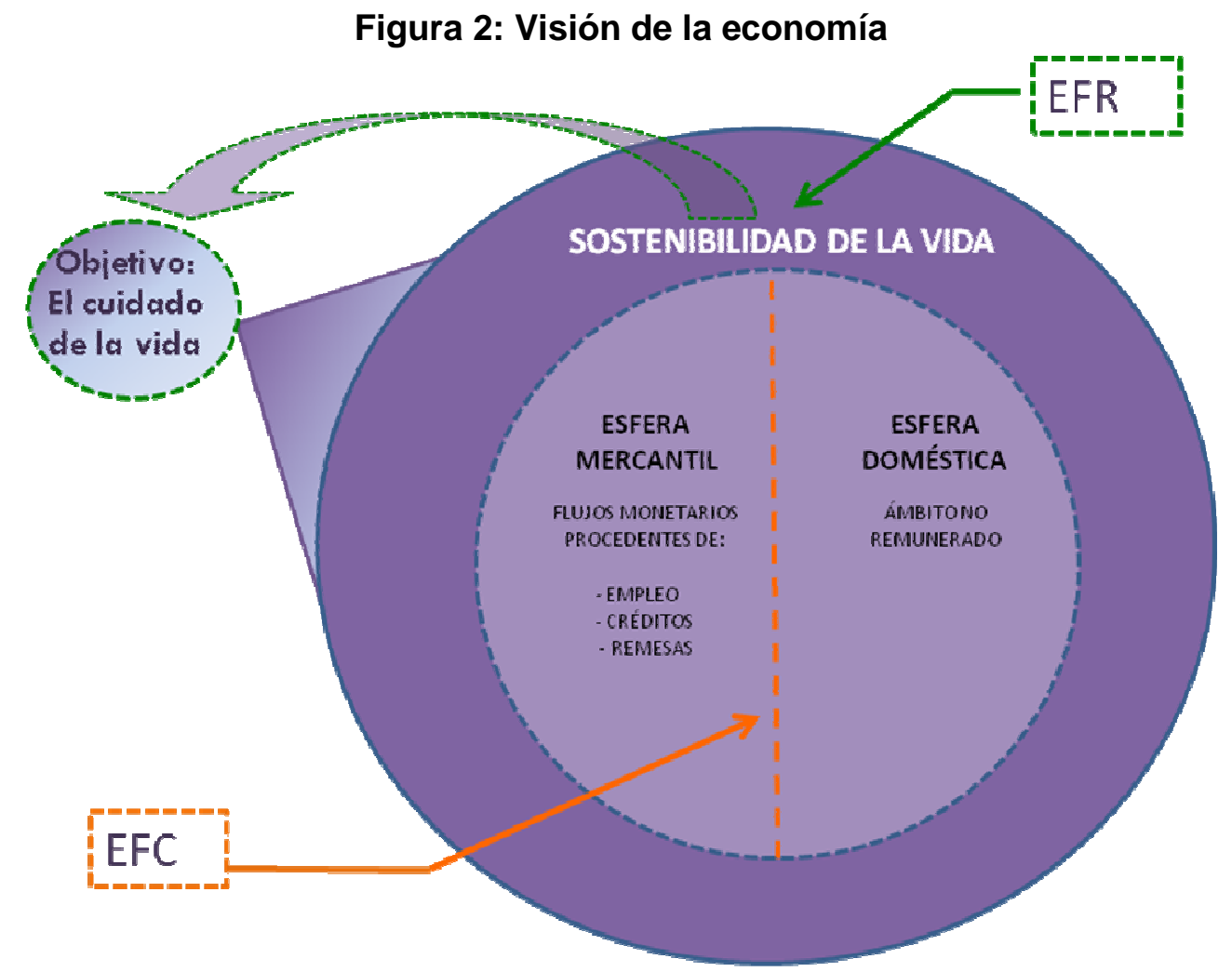

Fuente: Elaboración propia

\section{Metodologías de análisis}

La EFC propone suplir las metodologías económicas convencionales con métodos y conceptos nuevos, surgidos de las nuevas esferas que se han recuperado. Así se conforma una metodología diferente aunque derivada de la 
Agenjo, Astrid. Lecturas de la crisis en clave feminista: una comparación de la literatura en torno a los efectos específicos sobre las mujeres.

desarrollada para analizar las esferas monetizadas. De esta forma, las dificultades para captar la especificidad de las actividades no monetizadas se vuelven a poner en evidencia, teniendo que continuar valorándolas con referencia a la norma de los mercados. Un ejemplo claro es el intento de valorar el impacto de la crisis sobre el trabajo cuantificando el tiempo total que se dedica a ello. En este sentido el problema surge porque se aplica una noción lineal del tiempo, derivado de la noción de tiempo homogeneizable en unidades monetarias; pero como señala Carrasco "ni todos los tiempos son iguales ni son, por tanto, intercambiables" (Carrasco, 2001: 32).

Por su parte, la EFR propone una transformación radical de los métodos convencionales. Ejemplo de ello es la obtención de conceptos transversales como la sostenibilidad de la vida, el trabajo de cuidados, etc. con los que se pretende diluir los límites de la economía. Aunque apuestan por la interdisciplinariedad, no por ello descartan la utilización de herramientas convencionales; más bien se recurre a ellas de forma situada y ecléctica, "como una herramienta útil para un fin concreto y nunca como corsé prefijado" (Pérez, 2006: 13).

En este punto es necesario mencionar que el concepto de la doble presencia, utilizado en ambas corrientes, también es un ejemplo de concepto transversal. Éste pretende captar el proceso de generación de bienestar social identificando las interrelaciones entre las esferas de producción y reproducción. Como se comentó en su momento, lo que interesa a las autoras es comprender cómo las mujeres despliegan sus estrategias vitales en ambas esferas simultáneamente en un momento de crisis como el actual. Para ello consideran necesario integrar en el análisis las esferas del mercado, el Estado y los hogares, de ahí el hecho de incorporar elementos mercantiles como el trabajo asalariado, el acceso al crédito o las remesas; elementos procedentes de la esfera pública como los servicios sociales, la educación, la salud, etc.; y elementos procedentes de la esfera doméstica como los trabajos que se realizan de forma gratuita en el hogar (el trabajo doméstico y el trabajo de subsistencia). La diferencia fundamental entre las dos corrientes es que, mientras que en la EFC estos elementos conforman la parte central de su análisis, la EFR se fija en algunos de ellos sólo de forma estratégica para analizar los efectos específicos del declive económico y financiero.

\section{Efectos específicos de la crisis sobre las mujeres}

A raíz de la diferente metodología con la que se realiza el análisis de la crisis desde una y otra corriente, los resultados obtenidos en torno a los efectos específicos que ésta ha tenido sobre las mujeres también difieren.

En el análisis de la EFC la esfera mercantil no pierde la centralidad. Y esta es una cuestión fundamental porque esta forma particular de mirar la economía hace que se otorgue una importancia clave al impacto de la crisis sobre los aspectos como el trabajo asalariado, la autonomía financiera de las mujeres, el nivel de ingresos tras la pérdida de salario, la limitación del crédito, la disminución de remesas, etc. No obstante, también se presta una atención fundamental al impacto de la crisis sobre el ámbito doméstico, concluyendo que 
Agenjo, Astrid. Lecturas de la crisis en clave feminista: una comparación de la literatura en torno a los efectos específicos sobre las mujeres.

el principal efecto ha sido una intensificación en el tiempo de trabajo total de las mujeres.

Por tanto, con esta estrategia, la EFC se distancia de los esquemas sesgados de los enfoques androcéntricos que solo atendían a la parte monetizada de la realidad económica y logra eliminar la carga jerárquica de los elementos economía-no economía, trabajo-no trabajo. Si bien, aunque se incluya la esfera doméstica, ésta no importa en sí misma por su especificidad, sino que se recupera en tanto en cuanto se asimila al mercado: es decir, se descarta la existencia de actividades económicas distintivas de los hogares y se excluyen del análisis los aspectos personales y emocionales (afecto, cariño, culpabilidad, frustración...).

Por el contrario, en la EFR las personas (con sus cuerpos y sus identidades) sí adquieren una importancia central. Recordemos que en esta corriente el trabajo se entiende "como la práctica de creación y recreación de la vida y de las relaciones humanas" (Bosch et al, 2005: 331), una definición inclusiva que no puede visibilizarse dentro de los marcos convencionales. De ahí que esta corriente no pretenda establecer una frontera cerrada entre lo que es y no es economía y trabajo, sino que se opta por ofrecer conceptos transversales y "situados" en cada contexto concreto. En este caso se elige a los cuidados como punto de vista estratégico para comprender el efecto específico de la crisis sobre las mujeres, sacando a la luz una dimensión olvidada que les afecta de forma particular: la crisis de los cuidados.

El análisis que la EFR realiza en torno a los cuidados se basa en dos cuestiones fundamentales: en primer lugar, identificando qué agentes económicos participan en los procesos de satisfacción de necesidades humanas y cómo lo hacen; y en segundo lugar analizando cómo se resuelve el conflicto social de lógicas, es decir, en qué medida los mercados se han situado en el epicentro de la organización social y qué esferas y agentes absorben las tensiones de la coexistencia de dichas lógicas enfrentadas al asumir la responsabilidad última de que la vida continúe. Así, mientras que en la EFC la noción de "cuidados" se refiere al cuidado por parte de la familia, las instituciones gubernamentales y las no gubernamentales, en la EFR consideran como un todo, esto es, el hacerse cargo del mantenimiento de la vida y de la dependencia universal.

Esta mirada amplia de la EFR resulta fundamental para concebir la economía desde otro punto de vista que se centra en las personas y en sus necesidades; pero al mismo tiempo supone una visión más compleja y con mayores dificultades para analizar empíricamente. Un ejemplo de ello es la escasa atención que prestan a los efectos concretos de la crisis económica y financiera al respecto del trabajo asalariado, el acceso al crédito, las remesas, etc.

La siguiente figura pretende describir de forma esquemática los efectos que se identifican desde una y otra corriente: 
Agenjo, Astrid. Lecturas de la crisis en clave feminista: una comparación de la literatura en torno a los efectos específicos sobre las mujeres.

Figura 2: Efectos de la crisis

EFECTOS ESPECÍFICOS

SOBRE LAS MUJERES

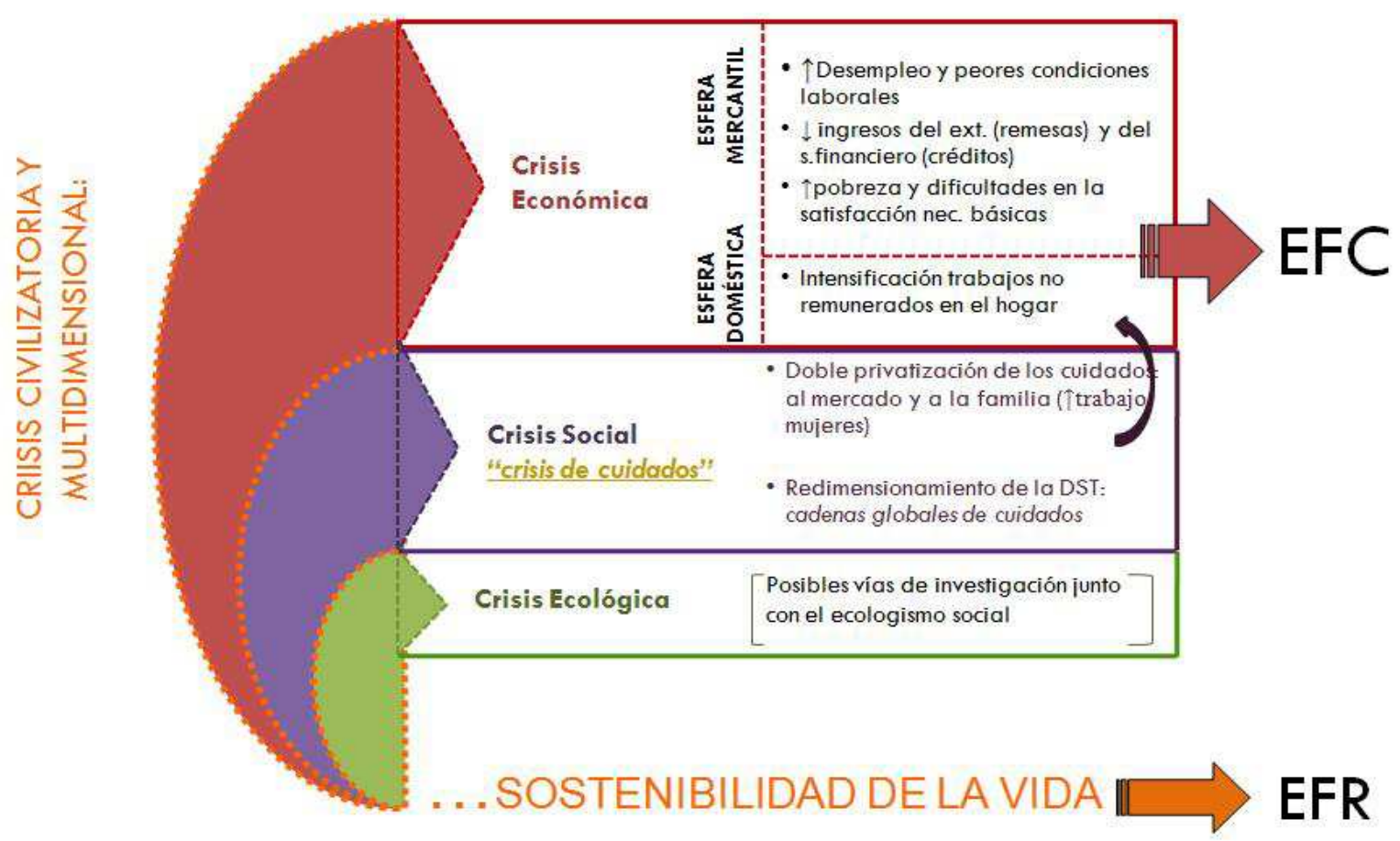

Fuente: Elaboración propia

\section{REFLEXIONES FINALES}

En su conjunto podemos concluir que, en el análisis de la crisis, la EFC se situaría en un plano más descriptivo, tratando de cuantificar los efectos diferenciados para mujeres y hombres en el plano monetizado y haciendo visible una parte importante del trabajo de las mujeres que permanece oculto en las estadísticas convencionales. Por su parte, la EFR opta por un análisis parcial y localizado, situando la raíz del problema de las mujeres en un nivel más profundo: el modelo de reparto de los cuidados que conforma la base del iceberg económico (a partir del cual, realizan diferentes propuestas sobre lo que debería ser la economía y hacia dónde deberían dirigirse los esfuerzos).

Por tanto, suponen dos niveles de discurso diferentes que dificultan su comparación, aunque no por ello son excluyentes, sino más bien complementarios. Es decir, representan una progresión argumentativa de la que se pueden obtener interesantes aportaciones. Por un lado, los datos cuantitativos que surgen de la EFC proporcionan una información empírica muy valiosa tanto para futuras investigaciones (por ejemplo, aquéllas que pretendan estudiar el impacto económico de la actual crisis sobre las mujeres y su comparación con otras anteriores), como para el planteamiento de respuestas políticas a la crisis a corto y medio plazo. En este sentido se plantean, por ejemplo, políticas salariales, políticas de redistribución de la renta, políticas de igualdad (referidas al tiempo de trabajo global y no solo al empleo formal: 
Agenjo, Astrid. Lecturas de la crisis en clave feminista: una comparación de la literatura en torno a los efectos específicos sobre las mujeres.

conciliación, permisos paternidad...), etc. No obstante, en estas páginas se considera que tales respuestas no suponen soluciones de raíz sino estrategias "de parcheo" frente a un sistema insostenible. Y precisamente, ésta es la principal crítica que se plantea al análisis de la EFC, en el sentido de que se pretenden conciliar dos esferas cuyas lógicas son de por sí opuestas e irreconciliables: el capital y la vida.

Por otro lado, las reivindicaciones de la EFR en torno a este conflicto social de lógicas, permiten plantear estrategias a más largo plazo encaminadas hacia una responsabilidad social en la sostenibilidad de la vida. Si bien en este texto se asume que ciertamente ése debe ser el objetivo a perseguir, también en este punto surge una reflexión esencial: ¿cómo se pueden materializar estas reivindicaciones de la EFR para que calen en la sociedad? ¿Con qué bazas contamos las mujeres para poner sobre la mesa del debate político las amenazas que supone el hecho de no resolver la crisis de los cuidados? Estas cuestiones plantean una importante barrera a la hora de diseñar acciones concretas en el mundo real. $Y$ si bien se propone que las reivindicaciones tendrían que partir de la redefinición de los trabajos y de una redistribución equitativa de los mismos entre mujeres y hombres, la clave es la siguiente: qué movimientos estratégicos son necesarios para poner esto en marcha.

Por tanto, consideramos que lo óptimo para la Economía Feminista es unir esfuerzos para encontrar las sinergias ocultas que permitan afrontar estas cuestiones y aprovechar las complementariedades que surgen de las dos corrientes, emprendiendo un diálogo con otras perspectivas críticas de la economía e incluso con otras disciplinas sociales a la hora de hacer frente a los retos que plantea esta crisis. 
Agenjo, Astrid. Lecturas de la crisis en clave feminista: una comparación de la literatura en torno a los efectos específicos sobre las mujeres.

\section{BIBLIOGRAFÍA}

Agarwal, B. (2004): "Challenging Mainstream Economics: Effectivness, Relevance and Responsibility", IAFFE Newsletter, 14(3), Octubre 2004, 26 , www.iaffe.org

Albelda, R. (1997): Economics and Feminism: Disturbances in the Field, Twayne Publishers, Nueva York.

Alberdi, I. (2009): "Dimensiones de género en la crisis económica y financiera mundial" ponencia llevada a cabo por la directora ejecutiva de UNIFEM en el Seminario Internacional "La igualdad de género en tiempos de crisis", Universidad del País Vasco, San Sebastián, 2 Septiembre de 2009.

Alles, M. (2000): Mujeres, trabajo y autoempleo. Otro camino frente al desempleo y el desarrollo de la carrera, Granica, Barcelona, Buenos Aires, México, Montevideo, Santiago.

Amoroso, M., Bosch, A. y Fernández, H. (2003): "Arraigadas en la tierra" en Dones i Treballs (2003), 71-96.

Amott, T. L. (1993): Caught in the Crisis: Women and the U.S. Economy Today, Monthly Review Press, Nueva York.

Antonopoulos, R. (2009): "The Current Economic and Financial Crises: A Gender Perspective, Working Paper no 562, the Levy Economics Institute of Bard College, Mayo 2009.

Bakker, I. (ed.) (1994): The Strategic Silence: Gender and Economic Policy, ZedBooks, Londres.

Balbo, L. (1978): "La doble presencia" en Borderías et al. (comp.) (1994), 50314.

Beasley, C. (1994): Sexual Economyths: Conceiving a Feminist Economics, St. Martin's Press, Singapore.

Benería, L. (1999): "El debate inconcluso sobre el trabajo no remunerado", Revista Internacional del Trabajo, 118 (3), 321-46.

Benería, L. (2004): "Introducción. La mujer y el género en la economía: un panorama general" en Villota, P. (ed.) (2004), Economía y género. Macroeconomía, política fiscal y liberalización. Análisis de su impacto sobre las mujeres, Icaria, Barcelona, 23-74.

Benería, L. y Sen, G. (1983): "Desigualdades de clase y género y el rol de la mujer en el desarrollo económico: implicaciones teóricas y prácticas", Mientras Tanto, 15, 91-112.

Benston, M. (1969): "The Political Economy of Women"s Liberation", Monthly Review, 21 (4), 13-27.

Bianchi, M. (1978): "Más allá del 'doble trabajo" en Borderías et al. (comp.) (1994), 491-502.

Bosch, A., Carrasco, C. y Grau, E. (2005): "Verde que te quiero violeta. Encuentros y desencuentros entre feminismo y ecologismo", en Tello, E. La historia cuenta, Barcelona, Ediciones EI Viejo Topo, pp. 321-346.

Borderías, C., Carrasco, C., y Alemany, C. (comp.) (1994): Las mujeres y el trabajo. Rupturas conceptuales, Icaria, Barcelona.

Borderías, C. y Carrasco, C. (1994): "Las mujeres y el trabajo: aproximaciones históricas, sociológicas y económicas" en Borderías, et al. (comp.) (1994), 15-109.

Braidotti, R. y Butler, J. (1997): "Feminism by Another Name. Interview" en Weed. y Schor (eds.) (1997), 31-67. 
Agenjo, Astrid. Lecturas de la crisis en clave feminista: una comparación de la literatura en torno a los efectos específicos sobre las mujeres.

Bryceson, D. y Vuorela, U. (1984): "Outside the Domestic Labour Debate: Towards a Theory of Modes of Human Reproduction", Review of Radical Political Economics, 16 (2/3), 137-66.

Buvinic, M. (2009): "The Global Financial Crisis: Assessing Vulnerability for Women and Children, Identifying Policy Responses", Interactive Expert Panel, Commission on the Status of Women Fifty-third session, New York, March 2009.

Cairó, G. y Mayordomo, M. (2005): Por una economía sobre la vida, Icaria, Barcelona.

Carrasco, C. (ed.) (1999), Mujeres y economía. Nuevas perspectivas para viejos y nuevos problemas, Icaria, Barcelona.

Carrasco, C. (2001a): Tiempos, trabajos y géneros, Universidad de Barcelona, Barcelona.

Carrasco, C. (2001b): "La sostenibilidad de la vida humana: ¿un asunto de mujeres?" en León, M (Comp.) (2003), Mujeres y Trabajo: cambios impostergables, Porto Alegre, OXFAM GB, Veraz Comunicaçao, p.11-49.

Carrasco, C. (ed.) (2003): Mujeres y economía, Icaria, Barcelona.

Carrasco, C. (2004), "Hacia nuevos indicadores de trabajo y género. Un problema mucho más que estadístico", en Villota, Paloma de (ed.), Globalización y desigualdad de género, Madrid: Síntesis, pp. 103-29.

Carrasco, C. (2006): "La paradoja del cuidado: necesario pero invisible", en Revista de Economía Crítica, marzo, 2006, pp.: 39-64.

Carrasco, C. (2009): "Mujeres, sostenibilidad y deuda social", en Revista de Educación, número extraordinario 2009, pp. 169-191.

Carrasco, C. (2010): "Prólogo" en Girón, A. (Coord.): Crisis económica: Una Perspectiva feminista desde América Latina. CLACSO, Caracas, 13-16.

Carrasco, C. (2011): "La economía del cuidado: planteamiento actual y desafíos pendientes", Revista de Economía Crítica ํำ11.

CSW (2009): "Perspectivas de género de la crisis financiera: Issues paper", 53은 periodo de sesiones, Nueva York, 2 a 13 de Marzo de 2009.

Delphy, C. (1970): Por un feminismo materialista. El enemigo principal y otros textos, laSal, Barcelona (1982).

Dijkstra, G. y Plantenga, J. (eds.) (1997): Gender and Economics. A European Perspective, Routledge, Londres, Nueva York.

Durán, M.A. (1999): "La base del iceberg (La contribución del trabajo no remunerado al mantenimiento de la economía española)", Mimeo.

Durán, M.A (2000): Si Aristóteles levantara la cabeza, Cátedra, Madrid.

Elson, D. (2002) "International Financial Architecture: A view from the kitchen" en Politica Femina, pp.26-37.

Ferber, M. A. y Nelson, J. A. (eds.) (1993a): Beyond Economic Man: Feminist Theory and Economics, University of Chicago Press, Chicago. Traducido como Más allá del hombre económico, Cátedra, la Universidad de Valencia y el Instituto de la Mujer, 2003.

Ferber M. A. y Nelson, J. A. (eds.) (2003): Feminist Economics Today. Beyond Economic Man, University of Chicago Press, Chicago.

Floro, M.S. (1999): "Double Day/Second Shift" en Peterson y Lewis (eds.) (1999), 136-42.

Folbre, N. y Hartmann, H. I. (1988): "La retórica del interés personal. Ideología y género en la teoría económica” en Carrasco (ed.) (1999), 91-124. 
Agenjo, Astrid. Lecturas de la crisis en clave feminista: una comparación de la literatura en torno a los efectos específicos sobre las mujeres.

Fórum de Política Feminista (2009): Feminismo ante la crisis, Fórum de Política Feminista, Madrid.

Gálvez, L. y Torres, J. (2009): "La crisis económica y sus alternativas: una perspectiva de género" en FORUM DE POLÍTICA FEMINISTA: Feminismo ante la crisis, Fórum de Política Feminista, Madrid.

Gálvez, L. y Torres, J. (2010): Desiguales, Icaria, Barcelona.

Gardiner, J. (1997): Gender, Care and Economics, MacMillan Press Ltd, Londres.

Gherardi, N. (2009): "El acceso al cuidado desde un enfoque de derechos: nuevos escenarios, nuevos actores", ponencia presentada en la Reunión de Especialistas Análisis de la crisis económica y financiera desde la perspectiva de género, México D.F, julio 2009.

Haraway, D. J. (1991): Ciencia, cyborgs, mujeres: la reinvención de la naturaleza, Cátedra e Instituto de la Mujer D. L., Madrid, Valencia (1995).

Harding, S. (1986): The Science Question in Feminism, Ithaca Cornell University Press, Nueva York.

Harding, S. (ed.) (1987): Feminism and Methodology: Social Sciences Issues, Indiana University Press, Bloomington.

Hartmann, H. (1979): "Un matrimonio mal avenido: hacia una unión más progresista entre marxismo y feminismo", Zona Abierta, 24, 85-113.

Hartmann, H. (1981): "The family as the locus of gender, class and political struggle. The example of housework" en Harding (ed.) (1987), 109-3.

Herrero, Y. (2009): "Crisis ecológica Aprender a vivir pisando ligeramente sobre la Tierra", Ecologistas en Acción.

Herrero, Y. (2010) "Cuidar: una práctica política anticapitalista y antipatriarcal" en TAIBO, Carlos (Dir.) Decrecimientos. Sobre lo que hay que cambiar en la vida cotidiana, Los Libros de la Catarata, Madrid.

Hewitson, G. (1999): Feminist Economics: Interrogating the Masculinity of Rational Economic Man, Northampton, Edward Elgar Pub, Massachusets.

Himmelweit, S. (1995): "The Discovery of "Unpaid Work": The Social Consequences of the Expansion of Work", Feminist Economics, 1(2), 1-19.

Humphries, J. (ed.) (1995): Gender and Economics, Edward Elgar Pub, Aldershot, Brookfield.

Jacobsen, J. P. (1994): The Economics of Gender, Blackwell Publishers, Cambridge.

Jain, D. y Elson, D. (2010): "Vision for a Better World: From Economic Crisis to Equality", PNUD Gender Team.

Kuiper, E. y Sap, J. (eds.) (1996): Out of the Margin, Feminist Perspectives on Economics, Routledge, Londres, Nueva York.

Larrañaga, M. (2009): "Mujeres, tiempos, crisis: combinaciones variadas" en Revista de Economía Crítica 8(2), pp. 113-120.

León, M. (2009): Más allá de la crisis, América Latina en movimiento no 441, ALAI, Quito.

Mies, M. (1996): "Globalisation of the Economy and Women's Work in a Sustainable Society", 6th International Interdisciplinary Congress on Women, Adelaide, Australia, 22-26 de Abril de 1996.

Montaño, S. y Milosavljevic, V. (2009): "La crisis económica y financiera. Su impacto sobre la pobreza, el trabajo y el tiempo de las mujeres", CEPAL, Santiago de Chile. 
Agenjo, Astrid. Lecturas de la crisis en clave feminista: una comparación de la literatura en torno a los efectos específicos sobre las mujeres.

Morton, P. (1971): "A Woman"s Work is Never Done" en Altbach, E. (ed.) (1971), From Feminism to Liberation, Schankman Publishing Co, Cambridge, Massachussets, 211-27.

Nelson, J. (1995): "Feminismo y economía" en Villota, P. (ed.) (2000), La política económica desde una perspectiva de género. La individualización de los derechos sociales y fiscales en la Unión Europea, Alianza, Madrid, 29-51.

Nelson, J. (1996): Feminism, Objectivity and Economics, Routledge, Londres.

OIT (2009) "Tendencias mundiales del empleo de las mujeres 2009". Ginebra, Organización Internacional del Trabajo.

OIT (2011): "Global economic crisis, gender and employment: The impact and policy response", Employment Working Paper, №74.

Pazos, M. (2009): Los mal llamados derechos de las mujeres en crisis", Revista Trasversales, nำ15 verano 2009.

Pérez, A. (2006): Perspectivas feministas en torno a la economía: el caso de los cuidados, Consejo Económico y Social, Madrid.

Pérez, A. (2009): "Feminismo anticapitalista, esa Escandalosa Cosa y otros palabros" ponencia presentada en las Jornadas Feministas Estatales, Granada, 2009.

Pérez, A. (2010): "Diagnóstico de la crisis y respuestas desde la economía feminista" en Revista de Economía Crítica 9(1), pp. 132-144.

Peterson, J. y Lewis, M. (eds.) (1999): The Elgar Companion to Feminist Economics, Edward Elgar Pub, Cheltenham, UK; Northampton, MA.

Picchio, A. (1992): "El trabajo de reproducción, tema central en el análisis del mercado laboral" en Borderías et al. (comp.) (1994), 451-502.

Picchio, A. (2001): "Un enfoque macroeconómico "ampliado" de las condiciones de vida" en CARRASCO (ed.) (2001), pp.15-37.

Picchio, A. (2005): "La economía política y la investigación sobre las condiciones de vida", en Cairó i Céspedes, G. y Mayordomo, M. (comps.), Por una economía sobre la vida. Aportaciones desde un enfoque feminista, Icaria, Barcelona, pp, 17-34.

PNUD y UNIFEM (2009): United Nations Conference on the World Financial and Economic Crisis and its Impact on Development, Joint Statement, 24-26 de junio.

Pujol, M. (1992): Feminism and Anti-Feminism in Early Economic Thought, Edward Elgar Pub, Londres.

Reid, M. G. (1934): Economics of Household Production, John Wiley, Nueva York.

Robeyns, I. (2000): "Is There A Feminist Economics Methodology?" octubre de 2000, http://www.ingridrobeyns.nl/Ac_publ.html

Rubery, J. (1988), Women and Recession, Routledge and Kegan Paul, Londres

Schneider, G. y Shackelford, J. (2001): "Economics Standards and Lists: Proposed Antidotes for Feminist Economists", Feminist Economics, 7 (2), 77-89.

Seguino, S. (2009): "The Global Economic Crisis and its Gender Implications", Interactive Expert Panel, Commission on the Status of Women Fifty-third session, New York, 2-13 marzo de 2009.

Tinker, I. (ed.) (1990): Persistent Inequalities: Women and World Development, Oxford University Press, New York and Oxford.

Vara, M.J. (Coord.) (2006): Estudios sobre género y economía, Akal, Madrid. 
Agenjo, Astrid. Lecturas de la crisis en clave feminista: una comparación de la literatura en torno a los efectos específicos sobre las mujeres.

Villota, P. (ed.) (1999): Globalización y género, Síntesis, Madrid.

Villota, P. (ed.) (2003): Economía y género, Icaria, Barcelona.

Walby, S. (2009): "Gender and the Financial Crisis", Paper for UNESCO Project on Gender and the Financial Crisis, Lancaster University.

World Bank (2009): The Global Financial Crisis: Assessing Vulnerability for Women and Children.

Young, I. M. (1980): "Socialist Feminism and the Limits of Dual Systems Theory", Socialist Review, 10 (2/3), Marzo-Junio. 